\title{
In silico drug repositioning of FDA-approved drugs to predict new inhibitors for alpha-synuclein aggregation
}

\author{
Sedighe Sadat Jafaripour ${ }^{1}$, Sajjad Gharaghani ${ }^{2 *}$, Elmira Nazarshodeh ${ }^{2}$, Shozeb Haider ${ }^{3}$, Ali \\ Akbar Saboury ${ }^{1 *}$ \\ ${ }^{1}$ Institute of Biochemistry and Biophysics, University of Tehran, Tehran, Iran \\ ${ }^{2}$ Laboratory of Bioinformatics and Drug Design, Institute of Biochemistry and Biophysics, University of \\ Tehran, Tehran, Iran \\ ${ }^{3}$ School of Pharmacy, University College London, UK \\ * Corresponding Authors: Ali Akbar Saboury (saboury@ut.ac.ir) \\ Sajjad Gharaghani (s.gharaghani@ut.ac.ir)
}

One of the hallmarks of Parkinson's disease (PD), a long-term neurodegenerative syndrome, is the accumulation of alpha-synuclein ( $\alpha$-syn) fibrils. Despite numerous studies and efforts, inhibition of $\alpha$ syn protein aggregation is still a challenge. To overcome this issue, we propose an in silico pharmacophore-based repositioning strategy, to find a pharmaceutical drug that, in addition to their defined role, can be used to prevent aggregation of the $\alpha$-syn protein. Ligand-based pharmacophore modeling was developed and the best model was selected with validation parameters including $72 \%$ sensitivity, $98 \%$ specificity and goodness score about 0.7 . The optimal model has three groups of hydrogen bond donor (HBD), three groups of hydrogen bond acceptor (HBA), and two aromatic rings (AR). The FDAApproved reports in the ZINC15 database were screened with the pharmacophore model taken from inhibitor compounds. The model identified 22 hits, as promising candidate drugs for Parkinson's therapy. It is noteworthy that among these, 10 drugs have been reported to inhibition of $\alpha$-syn aggregation or treat/reduce Parkinson's pathogenesis. This model was used to virtual screen ZINC, NCI databases, and natural products from the pomegranate. The results of this screen were filtered for their inability to cross the blood-brain barrier, poor oral bioavailability, etc. Finally, the selected compounds of two ZINC and NCI databases were combined and structurally clustered. Remained compounds were clustered in 28 different clusters, and the 17 compounds were introduced as final candidates.

Keywords: alpha-synuclein ( $\alpha$-syn); Aggregation inhibitors; Pharmacophore-based repositioning; 


\section{Introduction}

Parkinson's disease (PD) is a neurodegenerative disease, which has a direct relationship with aging. PD can be seen in patients with delayed voluntary movement. Studies showed that neurodegenerative diseases will become the second disease-related causes of human mortality after cardiovascular disease by 2040 (Valera and Masliah, 2016). Researchers have been trying to develop drugs for complementary treatment or at least for control of this disease (Kalia et al., 2013) (Naeem et al, 2018). A characteristic of PD is the accumulation of spherical insoluble bodies called the Lewy bodies around nerve cells, which filled with the aggregation of $\alpha-$ Synuclein ( $\alpha$-syn) proteins. $\alpha$-syn is an abundant presynaptic brain protein, which belongs to the family of Intrinsically disordered proteins or intrinsically disordered regions (IDPs or IDRs), (Breydo et al., 2012), that lack a rigid 3D structure under physiological conditions. These proteins adopt a variety of structural conformations upon interaction with different partners and play crucial biological roles in cellular processes (Daughdrill et al., 2005)(Uversky et al., 2008).

There is no doubt that strategy, which can inhibit or disrupt aggregation, can be effective routes for treatment or prevention of PD and thereby contributing to increased longevity of life. Thus the discovery of compounds that can serve as strong inhibitors and delay $\alpha-$ syn aggregation remains an important challenge. For this purpose, significant research has been done to identify $\alpha$-syn fibrillation inhibitor compounds. To the best of our knowledge, more than 60 small molecule inhibitors against $\alpha$-syn aggregation have been reported (Ardah et al., 2014a)(Caruana et al., 2011a)(Masuda et al., 2006b). Nevertheless, experimental studies to find successful candidate compounds are limited while conducting tests for all existing compounds is time-consuming and not cost-effective. Also, each of the reported compounds has substructures that further limit their application for therapeutic purposes.

Nowadays, virtual screening has become an essential part of the drug discovery process that endeavors to reduce time, costs, and removal of drug candidates with poor absorption, distribution, metabolism, elimination, and toxicity (ADMET) properties at the early stage before their synthesis. In general, this method tries to lead optimization by reducing laboratory tests. In rational drug design, the pharmacokinetic properties of compounds will be used to describe their inhibitory characteristics. The recent concept of 'polypharmacology', which proposes that drugs work mostly by acting on multiple targets, is being increasingly accepted (Macdonald et al., 2006)(Xie et al., 2012). Furthermore, polypharmacological features could help to find new uses of existing drugs via drug repositioning (Ashburn and Thor, 2004). Eventually, these approaches can help to find drugs for new targets, in addition to their well-established role. 
In this manuscript, we report a pharmacophore-based drug-repositioning method for drug discovery. To achieve this goal we built a pharmacophore model of $\alpha$-syn fibrillation inhibitors. This pharmacophore model used as a template to screen various databases such as NCI (290000 molecules), ZINC (Irwin and Shoichet, 2005) (270000 compounds), natural product compounds from pomegranate, a fruit that has remarkable antioxidant effects and FDA approved drugs ("Pomegranate and its Many Functional Components as Related to Human Health : A Review,” 2010).

\section{Materials and methods}

\section{Data collection}

To create a ligand-based pharmacophore model, we collected all $\alpha$-syn aggregation inhibitors that are publicly available. A dataset was obtained containing 60 reported inhibitor combinations belonging to various chemical structures, 8 clusters, using the Pharmacophore RDF-Code similarity implemented in ligand Scout and the cluster distance was set to 0.4 with average calculation method. This set has diverse activities from $61 \mathrm{~nm}-<80 \mu \mathrm{m}$. The structure of the molecules was taken from the PubChem. Compounds were divided into two groups: strong and weak, with an $I C_{50}$ value of about $10 \mu \mathrm{M}$ and those between 40-80 $\mu \mathrm{M}$, respectively (Masuda et al., 2006a) (S1, S2 ad S3).

\section{Pharmacophore model generation}

Pharmacophore can be modeled based on two methods i.e. structure-based or ligand-based (Angelbello et al., 2016). To create a structure-based model, the three-dimensional structure of $\alpha$-syn is required. The conformational space of IDPs is extremely challenging. This is because they lack a unique structure and in turn can adapt to different complexes in interaction with partners. In solution-state NMR, signals of the IDR in the interacting region becomes unrecognizable, while X-ray crystallography gives structural information on entirely ordered regions (Charlier et al., 2017). For this reason, we ignored the structure-based pharmacophore-model and explored the ligand-based method instead. Ligand-based pharmacophore model carried out using default settings of Ligand Scout 4.2 software available from InteLigand, GmbH, Vienna, Austria (http://www.inteligand.com/ligandscout)(Wolber and Langer, 2005a).

\section{Pharmacophore model validation}

The predictive performance of the models was evaluated by validation filters. The validation process usually helps to optimize or refine the model. A good model displays significant sensitivity and specificity. High sensitivity makes it possible to detect active compounds among the many inactive ones that are present in the group. The specificity reduces the error in distinguishing active compounds from 
the inactive. These two parameters are complementary and work in tandem. An ideal model has an optimal balance between these two parameters. The scoring function or the 'Fit scores' is also indicative of how functional groups of the compounds are matched with the pharmacophore model. The external validation approaches are test set/ decoy set and receiver operating characteristics (ROC) analysis is also considered as a signal-noise ratio.

In the test set method, 58 molecules with reported experimental activity values from $61 \mathrm{~nm}$ to $>80 \mu \mathrm{m}$ used to detect the predictive ability of the pharmacophore model (the results shown in Table 1). In the decoy set validation, a decoy database including 352 compounds generated by the virtual decoy set method (Wallach and Lilien, 2011). Physicochemical characteristics (molecular weight, hydrogen bond donor and acceptor count, number of rotatable bonds, the octanol/water partition coefficient) of the decoy were similar to the 18 reported active compounds, but spatial arrangements were different and calculated according to the Tanimoto coefficient (Wallach and Lilien, 2011).

\section{Database screening}

The model with the best scores in validation was used as a template for searching against various databases to find molecules that can potentially have an inhibitory effect. The screening was done using Ligand- Scout's default parameters: the scoring function, the retrieval mode, and the screening mode were set to "pharmacophore-fit", "Stop after first matching conformation" and "match all query features" respectively. The maximum number of omitted features was set to zero and check exclusion volume turned off. (Wolber and Langer, 2005b)(Temml et al., 2017).

\section{Drug-likeness selection and clustering of candidates}

At this stage, selected hits from NCI and ZINC (Irwin and Shoichet, 2005) databases were screened and covalent inhibitors, duplicate compounds, CNS (molecular mass $\leq 582 \mathrm{Da}$; the number of hydrogen bonding donors $\leq 3$, number of hydrogen bond acceptors $\leq 5$ and octanol-water partition coefficient $(\log \mathrm{P}) \leq 6)$ and "Pan Assay Interference Compounds" (PAINS) (false positive molecules) were identified and removed. The FAFdrug3 (Lagorce et al., 2015) online server (http://fafdrugs3.mti.univparis-diderot.fr) was engaged to check the described properties of candidate ligands.

\section{Dataset for drug repositioning}

The optimal pharmacophore model is used as a template for screening 1448 Food and Drug Administration approved (FDA-approved) drugs from the ZINC15 database (http://ZINC15.docking.org) (Sterling and Irwin, 2015). 
A molecular docking study was performed to investigate the binding affinity of the alpha-synuclein with the screened molecule. The 3D NMR structure of alpha-synuclein of Homo sapiens, 1XQ8, was taken from the protein data bank. We used to MTiAutoDock 1.0, a free online (http://bioserv.rpbs.univparis-diderot.fr/services/MTiOpenScreen/) and open-source protein-ligand docking tool that it is based on the AutoDock 4.2 and MGLTools. All HETATMs of the PDB structure were removed, hydrogen atoms were added and grid center and dimensions of binding sites were identified (Labbé et al., 2015). The top 10 ranked poses are given as output and the pose with minimum energy was detected.

\section{Results and discussion}

\section{Pharmacophore model generation}

The structures of $60 \alpha$-syn aggregation inhibitors were gathered from the literature (Caruana et al., 2011b)(Ardah et al., 2014b)(Masuda et al., 2006b). Ligands set conformers were produced using the default settings of OMEGA, while the RMSD threshold was set to 0.7. Ligand Scout was used to generating conformations, which built a maximum of 500 conformers per molecule. Pharmacophore models were created with different training set groups. Ligand Scout inserted common pharmacophore properties for the best-aligned solutions. 10 pharmacophore hypotheses were built with a tolerance scale of 1.0 and ranked following the Pharmacophore-Fit score (Gidaro et al., 2016). The applied scoring function was the "Pharmacophore-Fit score and Atom Overlap" and the chosen pharmacophore type was the "shared" pharmacophore features. The pharmacophore model was optimized by changing the size and number of features. Finally, the model with the training group including Gallic acid and Exifone (belong to two different structural clusters) was introduced as an optimal model and is illustrated in Fig. 1. Gallic acid is a known potent inhibitor of $\alpha$-syn aggregation, with inhibitory activity of about 90\% (Ardah et al., 2014b).

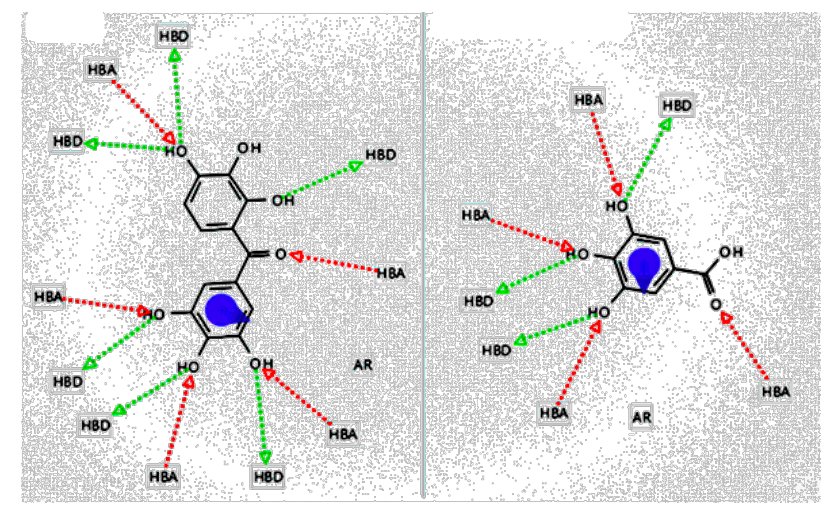

Figure 1. Chemical structures and Pharmacophore model of compounds Exifone(left) and gallic acid() (training set).

The features and the tolerance radii $(\AA)$ of the optimum model are shown in Fig. 2 and consist of two HBA, three HBD, and two AR. 


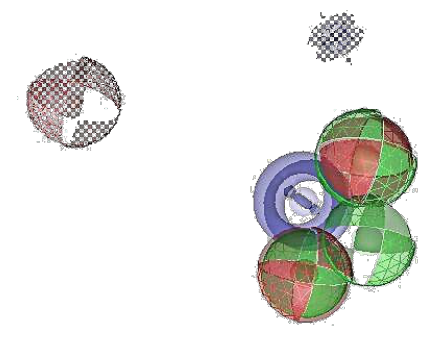

Figure2. Generated a ligand-based Pharmacophore model against alpha-synuclein aggregation that consists of three HBA (red), three HBD (green), two AR (blue) features.

\section{Evaluating the performance of the pharmacophore model}

This model has been validated by using test sets and decoy set methods, which display an extremely improved hit rate. Statistical parameters were calculated using the decoy set. These parameters include Total Hits (Ht), false negatives (FN), false positive (FP), goodness-of-hit score (GH), and enrichment factor (E-value) (Vuorinen et al., 2014). The statistical parameters obtained from the decoy test are listed in Table 1. The main parameters, which play a significant role in recognizing the ability of the pharmacophore models, are E-value and GH score.

Table 1. Statistical parameters obtained from decoy test

\begin{tabular}{clc}
\hline No & Parameters & Value \\
\hline 1 & Total Molecules in Database (D) & 370 \\
2 & Total Number of Actives in Database (A) & 18 \\
3 & Total Hits $(\mathrm{Ht})$ & 19 \\
4 & True positives $(\mathrm{TP})$ & 13 \\
5 & $\%$ Yield of actives $[=\{(\mathrm{TP} / \mathrm{Ht}) 100\}$ & $68.4 \%$ \\
6 & True negative $(\mathrm{TN})$ & 346 \\
7 & False negatives $(\mathrm{FN})=[\mathrm{A}-\mathrm{TP}]$ & 5 \\
8 & False positives $(\mathrm{FP})=[\mathrm{Ht}-\mathrm{TP}]$ & 6 \\
9 & Sensitivity $=\mathrm{TP} / \mathrm{A}$ & 0.72 \\
10 & Specificity $=$ TN/D-A & 0.98 \\
11 & Enrichment factor $((\mathrm{E}-\mathrm{value}))=\left\{(\mathrm{TP} \times \mathrm{D}) /\left(\mathrm{Ht}_{\mathrm{x}} \mathrm{A}\right)\right\}$ & 14.06 \\
12 & Accuracy $=(\mathrm{TP}+\mathrm{TN}) /(\mathrm{TP}+\mathrm{TN}+\mathrm{FP}+\mathrm{FN})$ & 0.97 \\
13 & Goodness of Hit Score $(\mathrm{GHScore}):\left[(\mathrm{TP} / 4 \mathrm{HtA})(3 \mathrm{~A}+\mathrm{Ht})\left(1 \_\left(\mathrm{Ht} \_\mathrm{TP}\right) /\left(\mathrm{D} \_\right.\right.\right.$A $\left.\left.)\right)\right]$ & 0.68 \\
\hline
\end{tabular}

The model with a GH score of $\sim 0.7$ is very good (Niu et al., 2014). The efficiency of each pharmacophore model was evaluated by a ROC curve, which compares sensitivity versus the specificity (Fig. 3). This efficiency is based on their ability to selectively obtain various active compounds from a large list of decoys or inactive against the selected targets. 


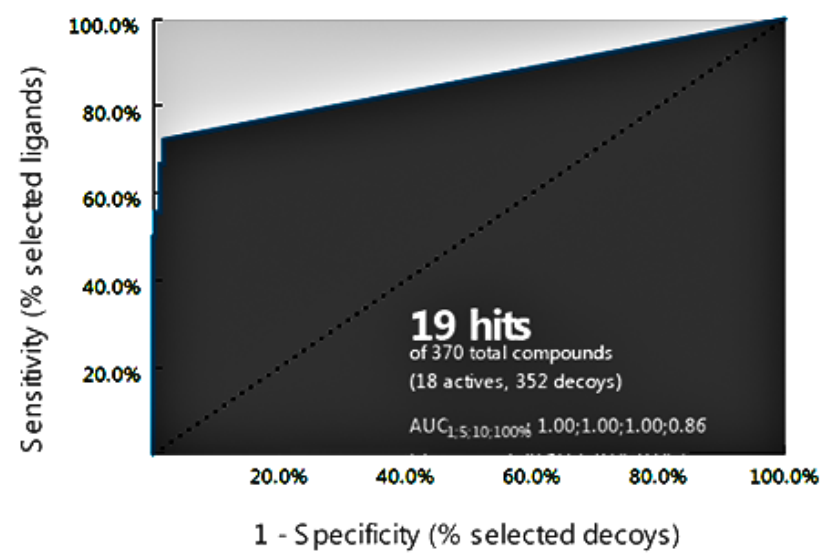

Figure 2. ROC Validation Curve for the Pharmacophore model

\section{Pharmacophore-based drug repositioning}

The ligand-based pharmacophore model identified 22 drugs that can be potential drug repositioning candidates (Table 2). The inhibitory effect of the drugs 2,3,4,15 and 20, which include Entacapone, Tolcapone, (Giovanni et al., 2010), Pyridoxine, Levodopa and Rifampicin (Török et al., 2016)(Li et al., 2004) respectively, have been previously used to study fibrillation of $\alpha$-syn. These drugs are used today to control the fibrillation of $\alpha$-syn and prevent the progression of Parkinson's disease. Drugs $8,9,10$, 14, and 16 (Epinephrine, Isoproterenol, Dopamine, Dobutamine, Nordefrin) are derivatives of dopamine that have shown inhibitory effect against $\alpha$-syn fibrillation (Conway et al., 2016). Interestingly, among these drug candidates, Carbidopa and Fenoldopam were suggested in previous studies to control the side effects of Parkinson's disease (Martino et al., 2016)(Twery, 1994). However, the effect of these drugs, as an inhibitor of fibrillation of $\alpha$-syn has not been reported. Also, it has recently been shown that asthma drugs can be considered for regulating and influencing the SNCA gene to control Parkinson's disease (Ridler, 2017). Interestingly, drugs No. 5, 6 (Salbutamol, and Formoterol) that were identified are two asthma drugs. Of the 22 candidates identified for drug repurposing, the inhibitory activity of 10 drugs has been previously reported. Therefore, the remaining candidate compounds, which include drugs 1, 5, 6, 7, 11,12, 13, 17, 18, 19, 21, and 22, can be potential inhibitors of $\alpha$-syn and also for the treatment of Parkinson's disease. 
Table 2.virtual screening of ZINC15 database with the pharmacophore model

\begin{tabular}{|c|c|c|c|c|c|c|}
\hline No. & Name & Fit Score & ZINC15_id & $\begin{array}{c}\text { Energy } \\
\text { (Kcal/mol)* } \\
\text { or } \\
\text { Docking } \\
\text { score* }\end{array}$ & Class & $\begin{array}{c}\text { Water } \\
\text { solubility } \\
(\mathrm{mg} / \mathrm{L})\end{array}$ \\
\hline
\end{tabular}

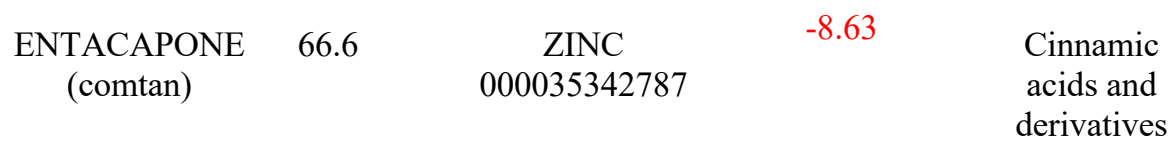

3

TOLCAPONE

(tasmar)

66.6

ZINC

000035342789

4

$\begin{array}{lcc} & \text { ZINC } \\ \text { PYRIDOXINE } & 65.67 & 000000049154\end{array}$

5

Salbutamol

(ALBUTEROL) 65.39

ZINC

000000007601
$-8.71$

Benzene and substituted derivatives

4000

-8.49 Benzazepines

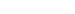




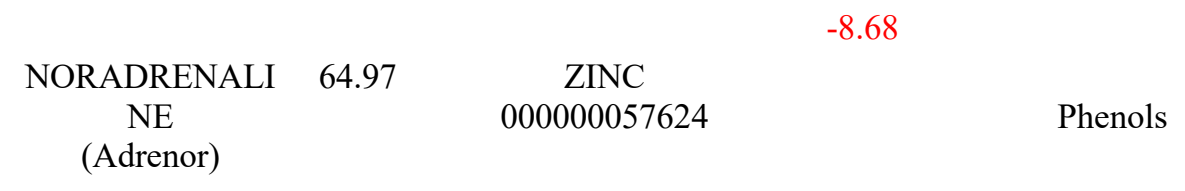

8

\begin{tabular}{|c|c|c|c|c|c|}
\hline & & $\begin{array}{c}\text { ZINC } \\
000000039089\end{array}$ & -8.68 & Phenols & $\begin{array}{c}\text { less than } \\
100\end{array}$ \\
\hline ZPINEPHRINE & 64.89 & & & Phenols & (at $64^{\circ} \mathrm{F}$ ) \\
\hline
\end{tabular}

9

$\begin{array}{ccccc} & \text { ZINC } & \\ \text { ISOPROTERENO } \\ \text { L } & 64.89 & 000000056652 & -8.69 & \text { Phenols }\end{array}$

10

$\begin{array}{lcccc}\text { DOPAMINE } & 64.82 & \text { ZINC } & -8.71 & \\ & & 000000033882 & & \text { Phenols }\end{array}$

11

\begin{tabular}{cccccc} 
& \multicolumn{3}{c}{ ZINC } & Phenylpropano \\
$\begin{array}{c}\text { METHYLDOPA } \\
\text { (RACEMIC) }\end{array}$ & 64.81 & 000000020255 & -8.73 & ic acids & $1 \mathrm{E}+004$
\end{tabular}

12

$\begin{array}{ccc}\text { TIPIRACIL } & 64.8 & \text { ZINC } \\ & & 000100032379\end{array}$

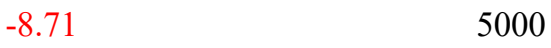

Diazines (warmed)

13

$\begin{array}{ccc}\text { CARBIDOPA } & 64.56 & \text { ZINC } \\ & & 000019168887\end{array}$
$-8.45$
Phenylpropano
ic acids

3.8

0.6 
15

$\begin{array}{llccc}\text { DOBUTAMINE } & 64.56 & \text { ZINC } & -8.69 & \text { Phenols }\end{array}$

$\begin{array}{ccc}\text { LEVODOPA } & 64.56 & \text { ZINC } \\ 000000895199\end{array}$

$-8.64$

Carboxylic acids and

(at $20^{\circ} \mathrm{C}$ ) derivatives

16

$\begin{array}{ccc}\text { NORDEFRIN } & 64.56 & \text { ZINC } \\ & & 000000034157\end{array}$

$-8.64$

Benzene and substitued derivaties

17

\begin{tabular}{|c|c|c|c|c|}
\hline L-Dops & 64.51 & $\begin{array}{c}\text { ZINC } \\
000002015035\end{array}$ & -8.67 & $\begin{array}{l}\text { Carboxylic } \\
\text { acids and } \\
\text { derivatives }\end{array}$ \\
\hline
\end{tabular}

18

CEFDINIR $\quad 64.44 \quad$ ZINC 000003927198

$-8.48$

Lactams

19

RIFAXIMIN

64.33

$-8.72$

Macrolactams

20

$\begin{array}{ccc} & & \text { ZINC } \\ \text { RIFAMPICIN } & 64.32 & 000169621223\end{array}$

$-8.59$

1400

(at $25^{\circ} \mathrm{C}$ ) 
METAPROTERE 64
NOL

22
ZINC

000000002273
TERBUTALINE $\quad 63.88$
ZINC

000000002281

* : binding affinity (docking) values of the hit compounds

\section{Database screening}

The results obtained from the mentioned analyses (model validation and pharmacophore-based drug repositioning) show that the validation of the model is sufficient enough for using it to screen other databases. Therefore, three databases were used for screening to identify new potential active compounds against $\alpha$-syn fibrillation. Hence, the validated pharmacophore model was applied as a template to search the NCI (https://www.cancer.gov/), ZINC (ZINC.docking.org/) databases (Table 3) and natural products from the Pomegranate fruit because of its unique characteristics ("Pomegranate and its Many Functional Components as Related to Human Health : A Review," 2010) (which contains 290981, 271910, and 22 molecules, respectively). Results of screening natural products from pomegranate fruit are shown in Table 4. 11 hits were identified from the pomegranate fruit compounds. Compounds No. 7, 8, and 11, have a fit score between 67-72. This can be considered a good fit score with the optimal pharmacophore model, have low conformers (Günther et al., 2006) and are therefore expected to be plausible inhibitors.

Table 3. Selected virtual hits from the ZINC and NCI subset databases

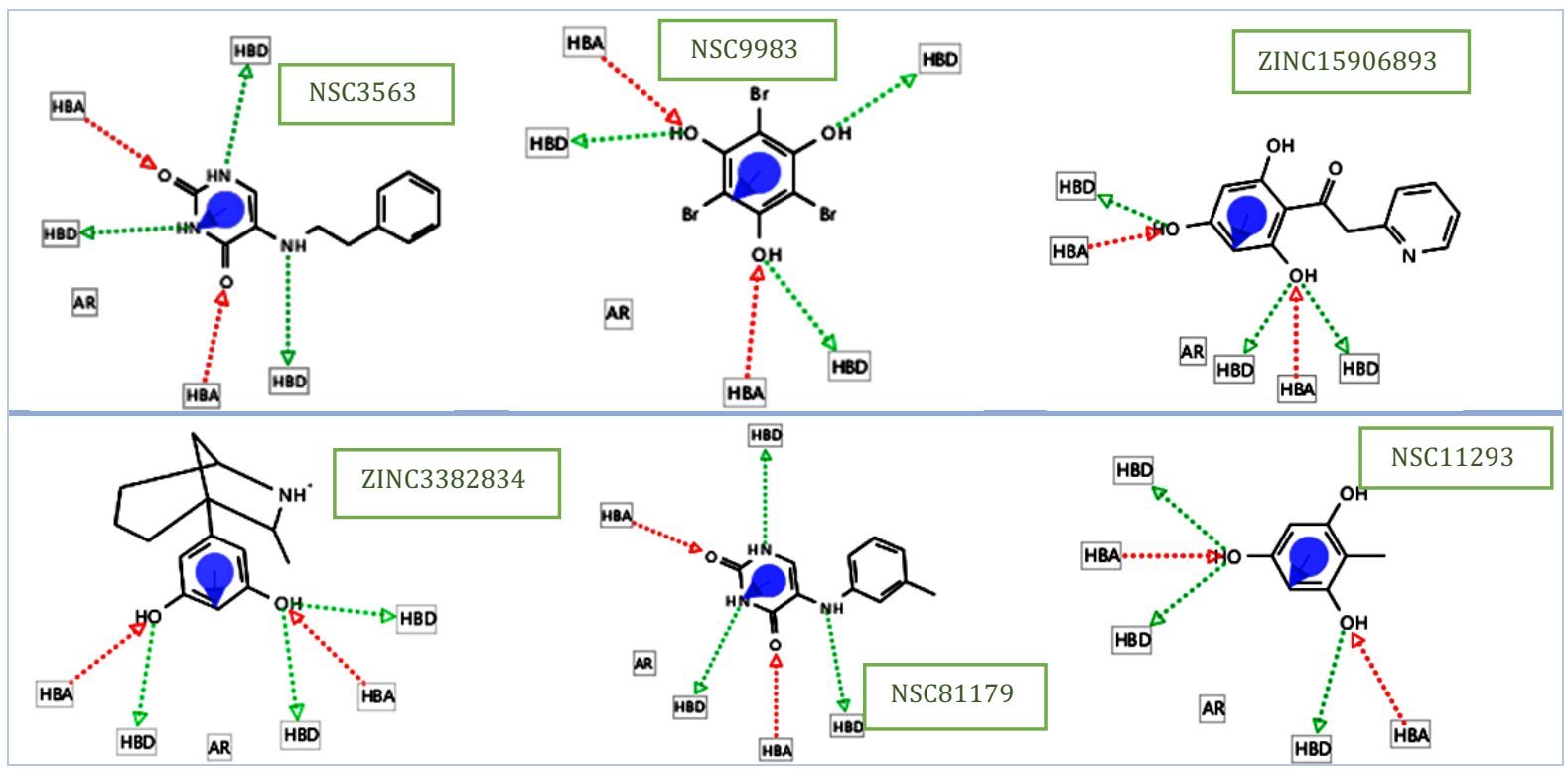




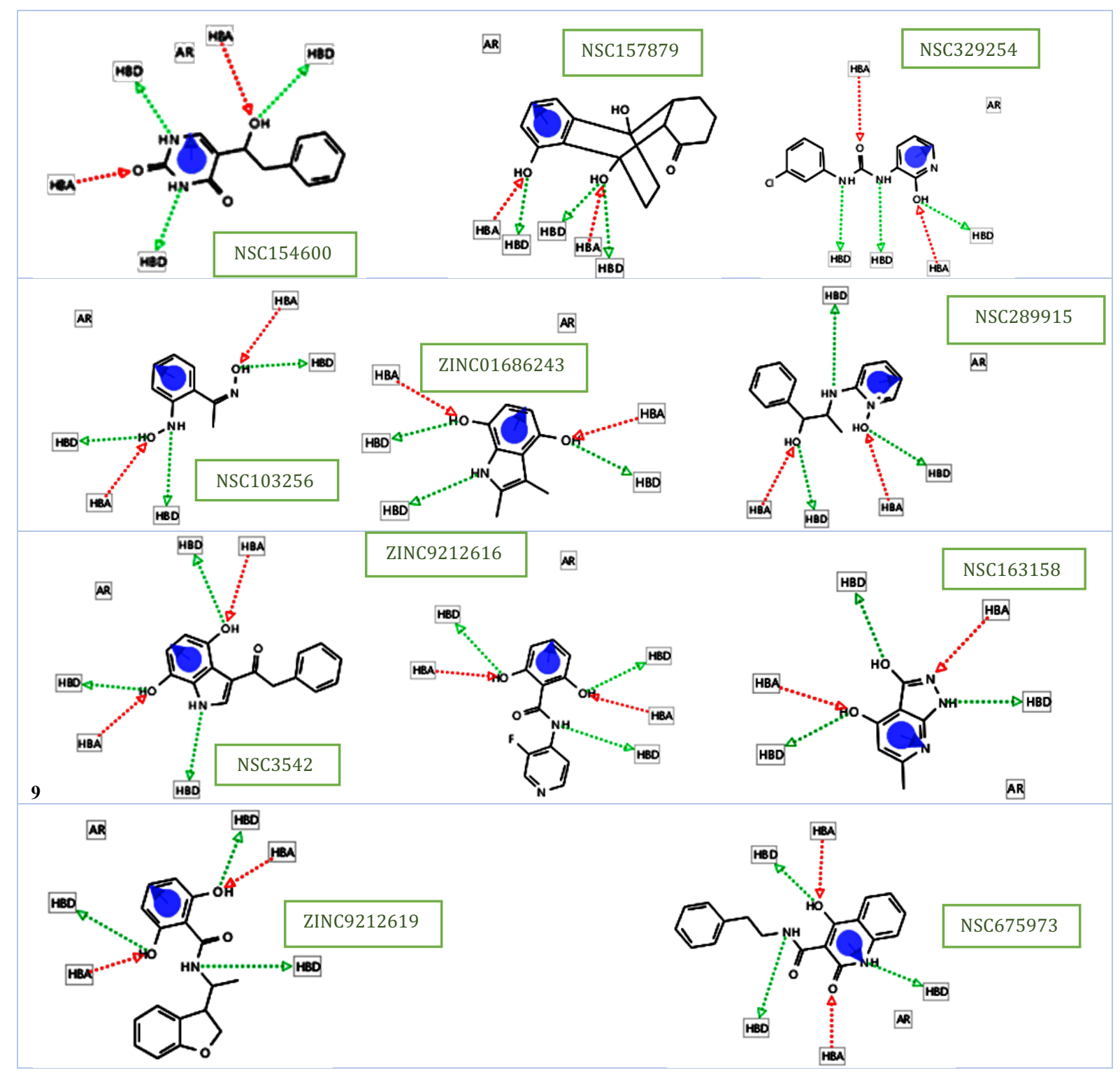

Table 4. Screening results of natural products from pomegranate

\begin{tabular}{llccc}
\hline No & compounds & $\begin{array}{c}\text { Energy } \\
(\text { Kcal/mol)* } \\
\text { or } \\
\text { Docking } \\
\text { score* }\end{array}$ & $\begin{array}{c}\text { Number of } \\
\text { conformers }\end{array}$ & $\begin{array}{c}\text { Pharmacophore } \\
\text { Fit Score }\end{array}$ \\
\hline $1 \quad$ Quercetin & -8.68 & 2 & 63.80 \\
2 & Epicatechin & -8.7 & 6 & 63.91 \\
3 & 5-O-Caffeoylquinic acid & -8.72 & 100 & 64.51 \\
4 & $\begin{array}{l}\text { Galloyl-HHDP-hexoside } \\
\text { (Corilagin) }\end{array}$ & -8.71 & 89 & 71.60 \\
5 & Brevifolin carboxylic acid & -8.7 & 2 & 67.65 \\
\hline
\end{tabular}




\begin{tabular}{rllll}
\hline \multicolumn{1}{c}{ V } & Valoneic acid bilactone & -8.64 & 10 & 67.62 \\
7 & Delphinidin-3-O-glucoside & -8.71 & 25 & 72.18 \\
8 & Cyanidin-3-O-glucoside & -8.68 & 31 & 63.77 \\
9 & Cyanidin-3-O-rutinoside & -8.63 & 98 & 63.76 \\
*: binding affinity (docking) values of the hit compounds & -8.71 & 15 & 72.23 \\
\cline { 2 - 4 }
\end{tabular}

Compound No. 10 (Punicalagin; Fig. 4) has been shown to possess strong antioxidant properties of pomegranate (Kulkarni and Aradhya, 2004)(Llorach and Tomás-barberán, 2003)(Rosenblat et al., 2013)(Ulkarni et al., 2007)(Seeram et al., 2005).

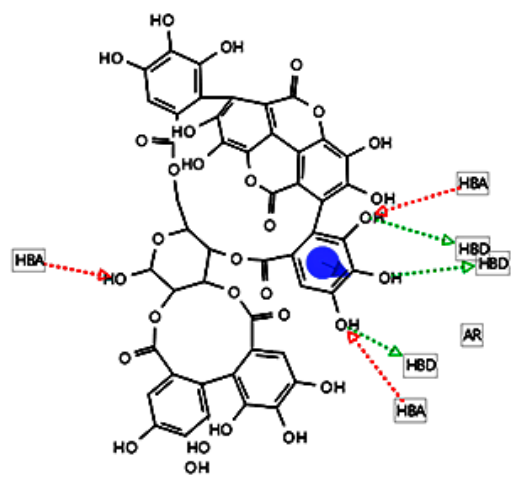

Figure4. Structure of Punigalagin fitted into the pharmacophore model

Additionally, the candidate compounds from ZINC and NCI were pre-filtered by the FAFdrug3 server (Yang et al., 2016). From the screening of ZINC and NCI databases, 44 and 53 compounds were selected respectively. Candidate compounds from these two databases were clustered into 28 different structural groups (Vuorinen et al., 2014). From each cluster, a member that has the best-fit score and compatibility with our pharmacophore model was selected. Finally, out of 28 candidate compounds, 18 compounds with up to 100 conformers were introduced as the final candidates (Günther et al., 2006). The results are shown in Table. 3. It is hoped that an experimental test will be done for these compounds in the future.

\section{Molecular docking}

To investigate the binding modes of the candidate compounds to the alpha-synuclein protein, molecular docking experiments were performed, which can evaluate the regional influence on biological activity. To find minimized affinity values, molecular docking of Exifone and Gallic acid, which was the 
compounds of the training set, was performed. The minimum free energy binding for these two compounds was -8.69 and $-8.68 \mathrm{Kcal} / \mathrm{mol}$, respectively. By performing molecular docking for the hit compounds of the ZINC15 and Pomegranate databases, the minimum free energy binding of these compounds was investigated (the results are shown in Tables 1 and 2). As it turns out, these minimum energies are close to the values obtained from the training set, and therefore confirm the model results.

\section{Conclusions}

In this study, we build a 3D ligand-based pharmacophore model that consists of three hydrogen bond donor (HBD), two hydrogen bond acceptor (HBA), and two aromatic rings (AR), with significant statistical parameters such as Sensitivity $=0.72$, Specificity $=0.98$ and GH-score $\sim 0.7$, which indicates the powerful ability of the model to pick out active molecules from the database. To perform drug repositioning, this model was used as a 3D query to screen the ZINC15 database. The inhibitory effect of a significant number of candidate drugs, such as Entacapone, Tolcapone, Pyridoxine, Levodopa, and Rifampicin was demonstrated in previous experimental studies. We then screened NCI, ZINC databases, and natural compounds from the pomegranate. Some compounds were proposed from each of these databases that could be potential inhibitors of $\alpha$-syn aggregation. Once again, the strength of the model was demonstrated by comparing the docking score of the selected ZINC and Pomegranate compounds with the training set. To best of our knowledge, this is the first report where a ligand-based pharmacophore model has been built and used to identify drugs for repositioning against $\alpha$-syn aggregation inhibition. Ultimately, we believe that this research will shed new light on better understanding pharmacophore features of potentially active compounds against the $\alpha$-syn aggregation. This study also certifies that there is a need to further in vitro and in vivo studies soon to develop potent drug inhibitors of the fibrillation of $\alpha$-syn for the treatment of synucleinopathy disorders like Parkinson's disease.

\section{Acknowledgment}

The financial support of the Research Council of the University of Tehran is acknowledged.

\section{References}

Angelbello, A.J., González, À.L., Rzuczek, S.G., Disney, M.D., 2016. Development of pharmacophore models for small molecules targeting RNA: Application to the RNA repeat expansion in myotonic dystrophy type 1 . Bioorganic and Medicinal Chemistry Letters 26, 57925796. https://doi.org/10.1016/j.bmcl.2016.10.037

Ardah, M.T., Paleologou, K.E., Lv, G., Khair, S.B.A., Kendi, A.K., Minhas, S.T., Al-Tel, T.H., AlHayani, A.A., Haque, M.E., Eliezer, D., El-Agnaf, O.M.A., 2014a. Structure activity relationship of phenolic acid inhibitors of ??-synuclein fibril formation and toxicity. Frontiers in Aging Neuroscience 6,1-17. https://doi.org/10.3389/fnagi.2014.00197 
Ardah, M.T., Paleologou, K.E., Lv, G., Khair, S.B.A., Kendi, A.K. Al, Minhas, S.T., Al-Tel, T.H., AlHayani, A.A., Haque, M.E., Eliezer, D., El-Agnaf, O.M.A., 2014b. Structure activity relationship of phenolic acid inhibitors of $\alpha$-synuclein fibril formation and toxicity. Frontiers in Aging Neuroscience 6,1-17. https://doi.org/10.3389/fnagi.2014.00197

Ashburn, T.T., Thor, K.B., 2004. Drug repositioning: identifying and developing new uses for existing drugs. Nature Reviews Drug Discovery 3, 673-683. https://doi.org/10.1038/nrd1468

Breydo, L., Wu, J.W., Uversky, V.N., 2012. $\alpha$-Synuclein misfolding and Parkinson's disease. Biochimica et Biophysica Acta - Molecular Basis of Disease 1822, 261-285. https://doi.org/10.1016/j.bbadis.2011.10.002

Caruana, M., Högen, T., Levin, J., Hillmer, A., Giese, A., Vassallo, N., 2011a. Inhibition and disaggregation of $\alpha$-synuclein oligomers by natural polyphenolic compounds. FEBS Letters 585 , 1113-1120. https://doi.org/10.1016/j.febslet.2011.03.046

Caruana, M., Högen, T., Levin, J., Hillmer, A., Giese, A., Vassallo, N., 2011b. Inhibition and disaggregation of ??-synuclein oligomers by natural polyphenolic compounds. FEBS Letters 585, 1113-1120. https://doi.org/10.1016/j.febslet.2011.03.046

Charlier, C., Bouvignies, G., Pelupessy, P., Walrant, A., Marquant, R., Kozlov, M., De Ioannes, P., Bolik-Coulon, N., Sagan, S., Cortes, P., Aggarwal, A.K., Carlier, L., Ferrage, F., 2017. Structure and Dynamics of an Intrinsically Disordered Protein Region That Partially Folds upon Binding by Chemical-Exchange NMR. Journal of the American Chemical Society 139, 12219-12227. https://doi.org/10.1021/jacs.7b05823

Conway, K.A., Rochet, J., Bieganski, R.M., Peter, T., Jr, L., 2016. Kinetic Stabilization of the $\alpha-$ Synuclein Protofibril by a Dopamine- $\alpha$-Synuclein Adduct Published by : American Association for the Advancement of Science Stable URL : http://www.jstor.org/stable/3085022 REFERENCES Linked references are available on JSTOR f. Science 294, 1346-1349.

Daughdrill, G.W., Pielak, G.J., Uversky, V.N., Cortese, M.S., Dunker, A.K., 2005. Natively Disordered Proteins.

Gidaro, M.C., Alcaro, S., Secci, D., Rivanera, D., Mollica, A., Agamennone, M., Giampietro, L., Concetta, M., Alcaro, S., Secci, D., Mollica, A., Agamennone, M., Giampietro, L., Gidaro, M.C., Alcaro, S., Secci, D., Rivanera, D., Mollica, A., 2016. Identification of new anti-Candida compounds by ligand-based pharmacophore virtual screening Identification of new antiCandida compounds by ligand-based pharmacophore virtual screening 6366. https://doi.org/10.3109/14756366.2016.1156103

Giovanni, S. Di, Eleuteri, S., Paleologou, K.E., Yin, G., Carrupt, P., Giovanni, S. Di, Eleuteri, S., Paleologou, K.E., Yin, G., Zweckstetter, M., Carrupt, P., Lashuel, H.A., 2010. Entacapone and Tolcapone, Two Catechol O -Methyltransferase Inhibitors, Block Fibril Formation of $\sqcup$ Synuclein and "ь-Amyloid and Protect against Amyloid-induced Toxicity * $\square$. https://doi.org/10.1074/jbc.M109.080390

Günther, S., Senger, C., Michalsky, E., Goede, A., Preissner, R., 2006. Representation of target-bound drugs by computed conformers : implications for conformational libraries 11, 1-11. https://doi.org/10.1186/1471-2105-7-293

Irwin, J.J., Shoichet, B.K., 2005. ZINC - A Free Database of Commercially Available Compounds for Virtual Screening ZINC - A Free Database of Commercially Available Compounds for Virtual Screening. J. Chem. Inf. Model 45, 177-182. https://doi.org/10.1021/ci049714

Kalia, L. V., Kalia, S.K., McLean, P.J., Lozano, A.M., Lang, A.E., 2013. A-Synuclein Oligomers and Clinical Implications for Parkinson Disease. Annals of Neurology 73, 155-169. https://doi.org/10.1002/ana.23746 
Kulkarni, A.P., Aradhya, S.M., 2004. Food Chemistry Isolation and identification of a radical scavenging antioxidant - punicalagin from pith and carpellary membrane of pomegranate fruit 87, 551-557. https://doi.org/10.1016/j.foodchem.2004.01.006

Labbé, C.M., Rey, J., Lagorce, D., Vavruša, M., Becot, J., Sperandio, O., Villoutreix, B.O., Tufféry, P., Miteva, M.A., 2015. MTiOpenScreen: A web server for structure-based virtual screening. Nucleic Acids Research 43, W448-W454. https://doi.org/10.1093/nar/gkv306

Lagorce, D., Sperandio, O., Baell, J.B., Miteva, M.A., Villoutreix, B.O., 2015. FAF-Drugs3: A web server for compound property calculation and chemical library design. Nucleic Acids Research 43, W200-W207. https://doi.org/10.1093/nar/gkv353

Li, J., Zhu, M., Rajamani, S., Uversky, V.N., Fink, A.L., 2004. Rifampicin Inhibits $\sqcup$-Synuclein Fibrillation and Disaggregates Fibrils 11, 1513-1521. https://doi.org/10.1016/j

Llorach, R., Tomás-barberán, F.A., 2003. Evaluation of the bioavailability and metabolism in the rat of punicalagin , an antioxidant polyphenol from pomegranate juice 28, 18-28. https://doi.org/10.1007/s00394-003-0396-4

Macdonald, M.L., Lamerdin, J., Owens, S., Keon, B.H., Bilter, G.K., Shang, Z., Huang, Z., Yu, H., Dias, J., Minami, T., Michnick, S.W., Westwick, J.K., 2006. Identifying off-target effects and hidden phenotypes of drugs in human cells 2, 329-337. https://doi.org/10.1038/nchembio790

Martino, T., Melchionda, D., Tonti, P., Francesco, V. De, 2016. Weight loss and decubitus duodenal ulcer in Parkinson' $s$ disease treated with levodopa - carbidopa intestinal gel infusion. Journal of Neural Transmission. https://doi.org/10.1007/s00702-016-1618-2

Masuda, M., Suzuki, N., Taniguchi, S., Oikawa, T., Nonaka, T., 2006a. Small Molecule Inhibitors of R -Synuclein Filament Assembly † 6085-6094. https://doi.org/10.1021/bi0600749

Masuda, M., Suzuki, N., Taniguchi, S., Oikawa, T., Nonaka, T., Iwatsubo, T., Hisanaga, S., Goedert, M., Hasegawa, M., 2006b. Small molecule inhibitors of alpha-synuclein filament assembly. Biochemistry 45, 6085-6094. https://doi.org/10.1021/bi0600749

Niu, M., Qin, J., Tian, C., Yan, X., Dong, F., Cheng, Z., Fida, G., Yang, M., 2014. Tubulin inhibitors : pharmacophore modeling, virtual screening and molecular docking 967-979. https://doi.org/10.1038/aps.2014.34

Pomegranate and its Many Functional Components as Related to Human Health : A Review, 2010. 9, 635-654. https://doi.org/10.1111/j.1541-4337.2010.00131.x

Ridler, C., 2017. Parkinson disease: Asthma drug could protect against PD. Nature Publishing Group 2017. https://doi.org/10.1038/nrneurol.2017.134

Rosenblat, M., Volkova, N., Aviram, M., 2013. ( punicalagin ) addition to statin , signi fi cantly protected against macrophage foam cells formation. Atherosclerosis 226, 110-117. https://doi.org/10.1016/j.atherosclerosis.2012.10.054

Naeem S, Akhtar S, Ali Z, Noori MY, Ahmed A, 2018. Pharmacophoric screening of newly synthesized isoniazid derivatives and their antimycobacterial activity and their antimycobacterial activity.

Seeram, N.P., Adams, L.S., Henning, S.M., Niu, Y., Zhang, Y., Nair, M.G., Heber, D., 2005. In vitro antiproliferative, apoptotic and antioxidant activities of punicalagin, ellagic acid and a total pomegranate tannin extract are enhanced in combination with other polyphenols as found in pomegranate juice 16, 360-367. https://doi.org/10.1016/j.jnutbio.2005.01.006

Sterling, T., Irwin, J.J., 2015. ZINC 15 - Ligand Discovery for Everyone. Journal of Chemical Information and Modeling 55, 2324-2337. https://doi.org/10.1021/acs.jcim.5b00559 
Temml, V., Garscha, U., Romp, E., Schubert, G., Gerstmeier, J., Kutil, Z., Matuszczak, B., Waltenberger, B., Stuppner, H., Werz, O., Schuster, D., 2017. Discovery of the first dual inhibitor of the 5-lipoxygenase-activating protein and soluble epoxide hydrolase using pharmacophore-based virtual screening. Scientific Reports 7, 6-13. https://doi.org/10.1038/srep42751

Török, N., Majláth, Z., Szalárdy, L., Vécsei, L., 2016. Expert Opinion on Investigational Drugs Investigational ? -Synuclein Aggregation Inhibitors : Hope For Parkinson's Disease Inhibitors of Alpha-Synuclein Aggregation : Hope For Parkinson' s Disease 3784. https://doi.org/10.1080/13543784.2016.1237501

Ulkarni, A.N.P.K., Ahal, H.S.M., Apoor, S.K., Radhya, S.M.A., 2007. In Vitro Studies on the Binding, Antioxidant, and Cytotoxic Actions of Punicalagin.

Uversky, V.N., Oldfield, C.J., Dunker, A.K., 2008. Intrinsically Disordered Proteins in Human Diseases: Introducing the $\mathrm{D}^{2}$ Concept. Annual Review of Biophysics 37, 215-246. https://doi.org/10.1146/annurev.biophys.37.032807.125924

Valera, E., Masliah, E., 2016. Therapeutic approaches in Parkinson's disease and related disorders. Journal of Neurochemistry 346-352. https://doi.org/10.1111/jnc.13529

Vuorinen, A., Engeli, R., Meyer, A., Bachmann, F., Griesser, U.J., Schuster, D., Odermatt, A., 2014. Ligand-based pharmacophore modeling and virtual screening for the discovery of novel $17 \beta$ hydroxysteroid dehydrogenase 2 inhibitors. Journal of Medicinal Chemistry 57. https://doi.org/10.1021/jm5004914

Wallach, I., Lilien, R., 2011. Virtual decoy sets for molecular docking benchmarks. Journal of Chemical Information and Modeling 51, 196-202. https://doi.org/10.1021/ci100374f

Wolber, G., Langer, T., 2005a. LigandScout : 3-D Pharmacophores Derived from Protein-Bound Ligands and Their Use as Virtual Screening Filters 160-169.

Wolber, G., Langer, T., 2005b. LigandScout: 3-D pharmacophores derived from protein-bound ligands and their use as virtual screening filters. Journal of Chemical Information and Modeling 45, 160-169. https://doi.org/10.1021/ci049885e

Twery MJ, Thompson LA, Walters JR., 1994. Intracellularly Recorded Response of Rat Striatal Neurons In Vitro to Fenoldopam and SKF 38393 Following Lesions of Midbrain Dopamine Cells 78, 67-78.

Xie, Lei, Xie, Li, Kinnings, S.L., Bourne, P.E., 2012. Novel Computational Approaches to Polypharmacology as a Means to Define Responses to Individual Drugs. https://doi.org/10.1146/annurev-pharmtox-010611-134630

Yang, Y., Zou, F., Zhao, L., Cheng, Y., Zha, X., Zhang, H., Zhou, J., 2016. Combined pharmacophore models as virtual screening protocol against BRD4(1) inhibitor. Medicinal Chemistry Research 25, 585-595. https://doi.org/10.1007/s00044-016-1513-2 